\section{Ehlers-Danlos syndrome: correlation with headache disorders in a young woman}

\author{
F. Di Palma ( $\varangle)$ • A.H. Cronin \\ Neurology Department, Headache Centre, \\ S. Anna Hospital, \\ Via Napoleona 60, I-22100 Como, Italy \\ e-mail: franco.dipalma@hsacomo.org \\ Tel.: +39-031-5855347 \\ Fax: +39-031-5855718
}

Published online: 15 December 2005

\begin{abstract}
Ehlers-Danlos syndrome is a rare inherited illness, which includes an autosomal dominant and also a recessive $\mathrm{X}$-linked variant. Its main clinical characteristic is a generalised connective tissue involving collagen and elastin, causing fragile and hyperextensible skin, loose jointedness and bruising. Many clinical subtypes are described, each of a different severity degree pattern. The correlation of this syndrome and headache disorders is rare. In this paper we describe the case of a young woman with Type II (less severe) Ehlers-Danlos Syndrome and headache.
\end{abstract}

Keywords Ehlers-Danlos syndrome - Migraine • Cervicogenic headache

\section{Case report}

We observed a 27-year-old female with Type II EhlersDanlos syndrome in the Headache Centre of our hospital. It is certainly inherited by a dominant autosomal pattern and may be very heterogeneous from a clinical point of view.

Our patient complained of joint tenderness with multiple orthopaedic troubles, including a lot of knee and shoulder subluxations. Also from a dermatologic point of view we observed a discrete extensibility (the patient became aware of this herself and was diagnosed with the syndrome after troublesome make-up procedures, e.g., while waxing her legs).

She came to the Headache Centre complaining of a severe pulsating unilateral headache, occurring 3 times per week, sometimes associated with typical visual aura. This symptomatology had lasted for 10 years and had worsened a year before, after neck trauma with whiplash syndrome. After this trauma the patient also reported a non-pulsating pain in the right side of the head, spreading to homolateral face and arm which overlapped with the previous symptomatology.

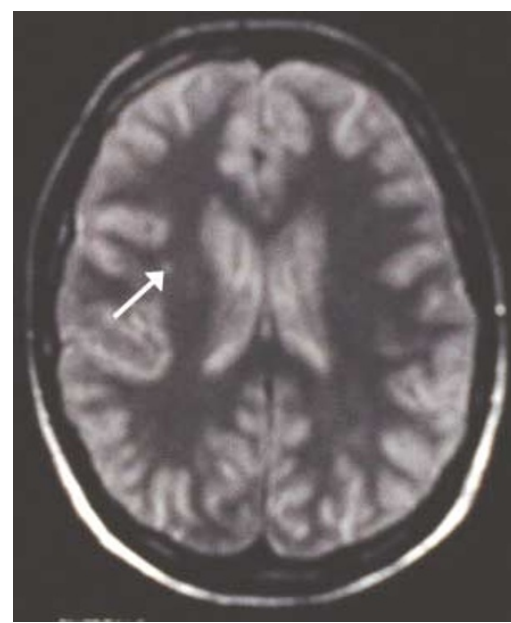

Fig. 1 Magnetic resonance imaging brain scan

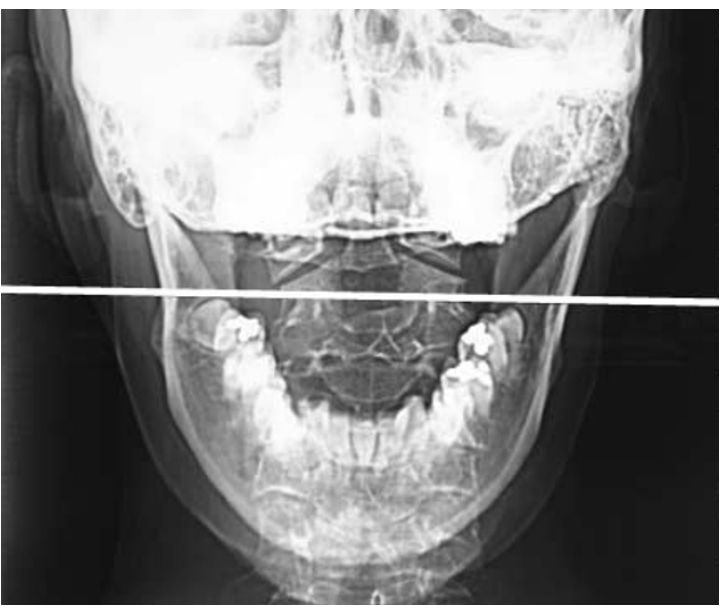

Fig. 2 Transoral X-radiogram

Our observation fulfils the IHS criteria of diagnosis for migraine without aura (1.1) and Cervicogenic Headache (11.2.1). It also fulfills the EHS Diagnostic Major Criteria for cervicogenic diagnosis.

She underwent a magnetic resonance imaging brain scan (evidence of small deep ischaemic lesions in both hemispheres) (Fig. 1) and a transoral X-radiogram (Fig. 2), which showed a dislocation of odontoid apophysis of $\mathrm{C} 2$ vertebra.

Prophylaxis with flunarizine was administered and migraine events decreased dramatically, but not those related to cervicogenic headache.

\section{Discussion}

Headache pathogenesis in Ehlers-Danlos is still uncertain. Sacheti et al. [1] conclude in their work that pain in neck 
and head could be sustained by connective tissue pathological relaxation. It is also known that disembryogenesis of the cortex can be present in Ehlers-Danlos syndrome, but the relationship between cortical neuron architectural malformation and possible cortical hyperexcitability has not yet been demonstrated.

Holzschuh et al. [2] demonstrated a low pulse index in all Ehlers-Danlos patients that underwent a transcranial Doppler, suggesting a vascular reactivity disorder that could play a role in migraine pathogenesis and as a stroke risk factor.

We reported this case because a link between EhlersDanlos syndrome and headaches is very rarely reported in the literature. Our review ranges from 1995 up to the present and we found only an 18-case cohort in Jacome in
1999 [3]. This confirms that headaches and particularly migraine are rare in Ehlers-Danlos syndrome.

\section{References}

1. Sacheti A, Szmere J, Bernstein B et al (1997) Chronic pain is a manifestation of the Ehlers-Danlos syndrome. J Pain Symptom Manage 14:88-93

2. Holzschuh M, Woertgen C, Browaski A(1996) Transcranial Doppler sonography in a patient with Ehlers-Danlos syndrome: case report. Neurosurgery 39:170-173

3. Jacome DE (1999) Headache in Ehlers-Danlos syndrome. Cephalalgia 19:765-766 